\title{
The Effect of Neck Flexion on Measurement of Spinal Bone Mineral Density and the Need to Apply Head Positioners During Dual-Energy X-Ray Absorptiometry
}

\author{
Mohsen Qutbi ${ }^{1}$, Sajad Ghanbari ${ }^{1}$, Mehdi Soltanshahi ${ }^{1}$, Saba Karami Gorzi $^{2}$, Yaser Shiravand ${ }^{1}$, and Shahla Ranji ${ }^{1}$ \\ ${ }^{I}$ Department of Nuclear Medicine, Taleghani Educational Hospital, School of Medicine, Shahid Beheshti University of Medical \\ Sciences, Tehran, Iran; and ${ }^{2}$ Science and Research Branch, Islamic Azad University, Tehran, Iran
}

Our purpose was to investigate any potential effect of neck flexion on measurement of spinal bone mineral density (BMD) through further reduction of spinal lordosis and whether it is necessary to apply a head positioner, in addition to a leg positioner, during dualenergy x-ray absorptiometry. Methods: Fifty-nine patients with no significant history of spinal disorders were recruited. A bone densitometry scan of the spine was obtained for all patients using a standard leg positioner in the supine position. Then, another scan of the spine was conducted using a small subnuchal cushion to flex the neck and, thus, straighten and minimize the lumbar lordosis. Parameters including the area, bone mineral content, BMD, and $T$ and $z$ scores for each lumbar vertebra (L1-L4) and for the total spine were extracted from the 2 scans and compared. Results: The mean age of the patients was 55.53 y $( \pm 11.86 \mathrm{y}) ; 53$ (89.83\%) were female and 6 (10.17\%) male. A statistically significant difference was found between corresponding values for area, BMD in L4, and total spine. The percentage change from a scan without a cushion to one with a cushion was $1.20 \%$ for $L 4$ and $0.58 \%$ for the total spine. The percentage BMD change was $-0.64 \%$ for $L 4$ and $-0.34 \%$ for the total spine. A change in diagnosis-from normal to osteopenia-occurred for only 1 patient. Conclusion: Use of a head positioner to flex the neck and thus minimize lumbar lordosis in dual-energy $x$-ray absorptiometry does not significantly affect the diagnosis or densitometric measurements from a clinical standpoint.

Key Words: bone mineral density; dual-energy x-ray absorptiometry; lordosis; head positioner

J Nucl Med Technol 2020; 48:340-343

DOI: 10.2967/jnmt.118.216275

\section{D} ual-energy x-ray absorptiometry is currently the unanimously accepted standard method for bone mineral density measurements. The standard procedural guidelines recommend that the lumbar spine be scanned with the patient supine and lying straight, with no axial rotation or lateral bending.

Received Mar. 14, 2020; revision accepted Jun. 6, 2020.

For correspondence or reprints contact: Mohsen Qutbi, Department of Nuclear Medicine, Taleghani Hospital, Yaman St., Velenjak, Tehran 1985711151, Iran.

E-mail: mohsen.qutbi@sbmu.ac.ir

Published online Jul. 24, 2020.

COPYRIGHT (c) 2020 by the Society of Nuclear Medicine and Molecular Imaging.
Furthermore, the scanning field of view should be centered on the spine (L1-L4) in both dimensions. It is important to minimize the curvature or lordosis of the lumbar spine by positioning the legs almost perpendicular to the trunk using a standard commercial leg positioner. This strategy encourages the lumbar vertebrae to lie at the same level as, or equidistant from, the radiation source or detector. Some recommend also using a head positioner to further minimize the lordosis and maximize patient comfort. Any type of spinal malpositioning may cause an over- or underestimation in the results (1-7). Spinal deformities, including lumbar scoliosis, increased lumbar lordosis, and even thoracic kyphosis, are important obstacles to achieving the correct positioning.

The impact of scoliosis has been investigated in previous studies $(8,9)$. Lordosis, another condition that may complicate interpretation of spine densitometry results, may be even more important because osteoporosis and spinal deformities (lordosis and scoliosis) are interrelated. A higher prevalence of spinal deformities has been demonstrated in osteoporosis patients than in individuals without osteoporosis $(10,11)$. In patients who have varying degrees of lordosis, the lumbar vertebrae do not lie horizontally on the scanning table (i.e., each is not equally distant from the $\mathrm{x}$ ray source and detector), even after a positioner is placed beneath the legs. This issue may create uncertainty in the densitometric results for the lumbar vertebrae.

One potential solution is to minimize lumbar lordosis by flexing the neck. When a subnuchal cushion is used, the lumbar vertebrae lie as level as possible on the scanning table, as well as being positioned more consistently from scan to scan. However, the extent of any possible influence on BMD, T scores, $z$ scores, and, thus, the final diagnosis has not been adequately investigated. To perform such an investigation, we scanned patients using the standard method (i.e., use of solely a leg positioner) and then repeated the scans with the addition of a head positioner.

\section{MATERIALS AND METHODS}

Fifty-nine patients, $53(89.83 \%)$ female and 6 (10.17\%) male, with no history of any cervical or lumbar spine disorders, including cervical spondylosis, significant lumbar lordosis, and previous operative 
procedures or prior trauma, were consecutively selected and then included in the study after they had given written informed consent. Patients younger than $18 \mathrm{y}$ or older than $80 \mathrm{y}$ were excluded because no reference database is available for statistical comparisons. The study was approved by the School of Medicine Ethics committee.

A bone densitometry scan of the spine was performed using the standard method, that is, placing a standard commercial positioner beneath the legs of the supine patient, as shown in Figure 1A. Then, without significantly changing the patient's position, another scan was performed with the addition of a small cushion ( $8 \mathrm{~cm}$ high) beneath the neck (Fig. 1B). Afterward, the hip scan was performed according to the standard protocol (from the proximal femur in internal rotation, with the foot of the index limb strapped to the standard positioner). A QDR series densitometry scanner (Hologic) with a standard manufacturer-designed leg positioner was used for all scans. Scans showing significant focal abnormalities in the L1-L4 vertebrae were excluded from the study.

All images were analyzed automatically, with minimum interaction by the operator, using the standard software provided by the manufacturer. If there were errors in region-of-interest placement or bone mapping, manual corrections were made. BMD (g/ $\mathrm{cm}^{2}$ ), $\mathrm{T}$ scores, and $z$ scores for each lumbar vertebra (L1-L4), for the total spine, and for the proximal femur were extracted from the 2 scans (before and after application of the subnuchal cushion). The corresponding data were compared by paired-sample $t$ testing using the SPSS software for statistical analysis. The significance level was set at 0.05 .

The diagnosis was based on the mineral density results for the lumbar spine and proximal femur (neck and total area). For menopausal women and men older than $50 \mathrm{y}$, we used the World Health Organization classification (in which a $\mathrm{T}$ score $\geq-1$ indicates normal findings, a $\mathrm{T}$ score between -1 and -2.5 indi-

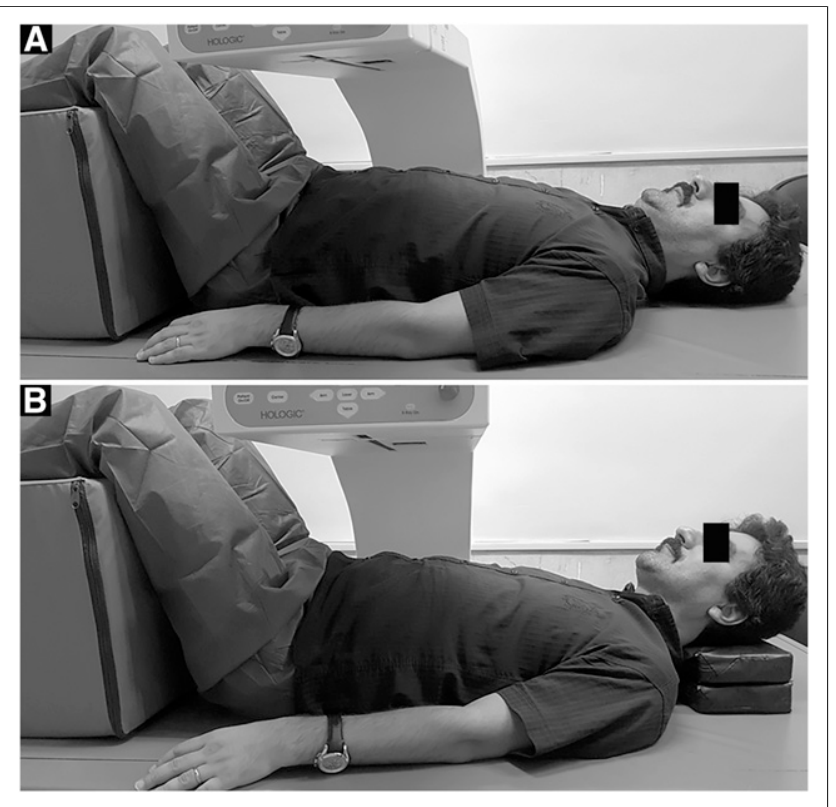

FIGURE 1. (A) Patient on scanning table with leg positioner beneath legs. (B) Without changing position of patient, cushion is placed beneath neck to achieve sufficient neck flexion. cates osteopenia, and a $\mathrm{T}$ score $\leq-2.5$ indicates osteoporosis). For premenopausal women and men younger than $50 \mathrm{y}$, we used the International Society for Clinical Densitometry classification (in which a $\mathrm{T}$ score $\leq-2$ is below the expected range for age and a $\mathrm{T}$ score $>-2$ is within the expected range for age). Changes in the diagnosis were expressed as numbers and percentages. Finally, the diagnoses derived from the 2 scans were tested for agreement to derive the $\kappa$-coefficient.

\section{RESULTS}

The demographics and densitometric characteristics of the 59 subjects are presented in Table 1. Area, bone mineral content, BMD, $\mathrm{T}$ scores, and $z$ scores are presented in Table 2. The error bar plots of area, bone mineral content, and BMD are presented in Figure 2.

A gradual increase in area, bone mineral content, and BMD was seen from L1 to L4. A statistically significant difference was found between corresponding values for area, $\mathrm{BMD}$, and $\mathrm{T}$ score in L4 and total spine. The difference in bone mineral content in total spine for both scans was not statistically significant. In L4 and total spine, the area was higher for the scan performed with a cushion, and conversely, the BMD was higher for the scan without a cushion. The percentage change from the scan without a cushion to that with a cushion was $1.20 \%$ and $0.58 \%$ for L4 and total

TABLE 1

Patient Characteristics

\begin{tabular}{lc}
\hline \multicolumn{1}{c}{ Description } & Data \\
\hline Age $(y)$ & $55.77( \pm 10.41)$ \\
Female & $53.33( \pm 22.29)$ \\
Male & $55.53( \pm 11.86)$ \\
Total & $18-76$ \\
Range & \\
Sex & $53(89.83 \%)$ \\
Female & $6(10.17 \%)$ \\
Male & $66.15( \pm 10.01)$ \\
Weight $(k g)$ & $159.83( \pm 6.88)$ \\
Height $(c m)$ & $26.00( \pm 4.23)$ \\
Body mass index & \\
Status & $8(13.6 \%)$ \\
Premenopausal female & $45(76.3 \%)$ \\
Menopausal female & $2(3.4 \%)$ \\
Male under $50 \mathrm{y}$ & $4(6.8 \%)$ \\
Male over $50 \mathrm{y}$ & \\
Femoral neck & $4.62( \pm 0.47)$ \\
Area & $0.701( \pm 0.12)$ \\
BMD & $-1.4( \pm 1.0)$ \\
T score & $-0.3( \pm 1.1)$ \\
$z$ score & \\
Total femur & $32.87( \pm 3.84)$ \\
Area & $0.818( \pm 0.11)$ \\
BMD & $-1.1( \pm 0.9)$ \\
T score & $-0.3( \pm 1.0)$ \\
$z$ score & \\
\hline
\end{tabular}

Qualitative data are numbers followed by percentages in parentheses; continuous data are mean $\pm \mathrm{SD}$. Total $n=59$. 
TABLE 2

Densitometric Measurements for Total Spine and L1-L4 Vertebrae

\begin{tabular}{|c|c|c|c|c|c|}
\hline \multirow[b]{2}{*}{ Vertebra } & \multicolumn{4}{|c|}{ Results } & \multirow[b]{2}{*}{$P$} \\
\hline & Without cushion & With cushion & Mean difference & 95\% confidence interval & \\
\hline \multicolumn{6}{|l|}{ Area } \\
\hline L1 & $12.24( \pm 1.49)$ & $12.32( \pm 1.52)$ & $-0.08( \pm 0.48)$ & $-0.20-0.05$ & 0.214 \\
\hline L2 & $13.80( \pm 1.69)$ & $13.74( \pm 1.54)$ & $0.06( \pm 0.51)$ & $-0.07-0.19$ & 0.355 \\
\hline L3 & $14.88( \pm 1.86)$ & $14.97( \pm 1.71)$ & $-0.08( \pm 0.42)$ & $-0.19-0.03$ & 0.135 \\
\hline L4 & $15.80( \pm 1.84)$ & $15.99( \pm 1.88)$ & $-0.19( \pm 0.54)$ & -0.33 to -0.05 & $0.009^{\star}$ \\
\hline Total & $56.73( \pm 6.37)$ & $57.06( \pm 6.14)$ & $-0.33( \pm 0.78)$ & -0.53 to -0.13 & $0.002^{*}$ \\
\hline Bone mineral content (total) & $49.96( \pm 10.22)$ & $50.09( \pm 10.16)$ & $-0.13( \pm 0.87)$ & $-0.36-0.09$ & 0.245 \\
\hline \multicolumn{6}{|l|}{ BMD } \\
\hline L1 & $0.774( \pm 0.14)$ & $0.774( \pm 0.15)$ & $0.000( \pm 0.02)$ & $-0.005-0.005$ & 0.906 \\
\hline L2 & $0.868( \pm 0.14)$ & $0.864( \pm 0.14)$ & $0.004( \pm 0.02)$ & $-0.001-0.009$ & 0.108 \\
\hline L3 & $0.905( \pm 0.14)$ & $0.902( \pm 0.14)$ & $0.003( \pm 0.02)$ & $-0.001-0.007$ & 0.189 \\
\hline L4 & $0.940( \pm 0.14)$ & $0.935( \pm 0.14)$ & $0.006( \pm 0.02)$ & $0.001-0.010$ & $0.011^{*}$ \\
\hline Total & $0.878( \pm 0.13)$ & $0.875( \pm 0.13)$ & $0.003( \pm 0.01)$ & $0.001-0.006$ & $0.018^{\star}$ \\
\hline T score (total) & $-1.57( \pm 1.2)$ & $-1.60( \pm 1.2)$ & $0.03( \pm 0.1)$ & $0.00-0.05$ & $0.046^{\star}$ \\
\hline z score (total) & $-0.42( \pm 1.3)$ & $-0.45( \pm 1.3)$ & $0.03( \pm 0.1)$ & $-0.00-0.05$ & 0.096 \\
\hline
\end{tabular}

*Statistically significant $P$ value.

spine, respectively. The percentage change was $-0.64 \%$ for L4 and $-0.34 \%$ for total spine. For the scan without a cushion, the results were normal in $11(18.6 \%)$, osteopenia in 19 (32.2\%), osteoporosis in 19 (32.2\%), within the expected range for age in $9(15.3 \%)$, and below the expected range for age in $1(1.7 \%)$. For the scan with a cushion, the results were normal in $10(16.9 \%)$, osteopenia in $20(33.9 \%)$, osteoporosis in $19(32.2 \%)$, within the expected range for age in $9(15.3 \%)$, and below the expected range for age in $1(1.7 \%)$. A change in diagnosis - from normal to osteopenia-occurred in only 1 patient $(1.7 \%)(\kappa=0.977)$.

\section{DISCUSSION}

Technical issues have an important effect on bone mineral densitometric measurements. Proper and consistent positioning is one such issue. This factor is highly important not only for obtaining accurate results but also for achieving serial scans comparable and thus suitable for decision making. The spine, because of the high proportion of trabecular tissue in its composition, is an indispensable part of densitometric measurements. But since there is a variable degree of lordosis, strategies should be adopted to flatten the lumbar spine as much as possible. Otherwise, intra- or interpatient uncertainty may arise.

Using a leg positioner to flex the hip joint up to $90^{\circ}$ is a routine part of the procedure, but in most patients some degree of lordosis may persist. In such situations, flexing the neck by placing a cushion beneath it may be help flatten the spine on the scanning table. Some manufacturers may supply a dedicated head positioner to routinely position the head during scanning, but the effect of using such a positioner is uncertain and has not been much investigated. The 8 -cm-high cushion we applied seemed to be sufficient to reduce the remaining lordosis after hip flexion, producing a statistically significant difference in the area and BMD of the L4 vertebra and the total spine.
The fact that no difference in bone mineral content was found suggests that the changes in BMD could be a function of area, but the changes were remarkably low in absolute values. Of the 59 individuals recruited for the study, only one showed a single-level change in diagnosis (i.e., from normal to osteopenia). This degree of potential error is considerably lower than that from spinal malpositioning and scoliosis $(2,3,9)$. In a study by Pavlovic et al. (8), the BMD of the spine was measured in patients with thoracic kyphosis and lumbar lordosis. The investigators concluded that the BMD decreases as the degree of curvature in the thoracic or lumbar spine increases. However, the correlation between the measured curvature and the spine BMD was weak to moderate, and these findings were the opposite of ours.

The concept of least significant change should also be considered when comparing serial scans such as those in our study. The rate of least significant change varies among centers, but an overall rate of $3 \%-4 \%$ is accepted and lower rates cannot be considered statistically significant $(12,13)$. In addition, this rate is much higher than those for spinal BMD in this study.

All the above findings may not lay a solid foundation to recommend the routine use of head positioners. However, the effect on patient comfort from raising the arms during scanning may be beneficial.

In the present study, we practiced methods to reduce lumbar lordosis in patients with a normal degree of lordosis or at least without significant abnormality. However, the degree of lordosis was not assessed or measured, either qualitatively or quantitatively, before the first and second scans, although there exist methods for measurement of spinal curvature (14). The flexibility or rigidity of the perispinal soft tissues and, therefore, lordosis varies among patients. The effect size may be diminished by the fact that there is a pool of patients with a nearly flattened spine after 


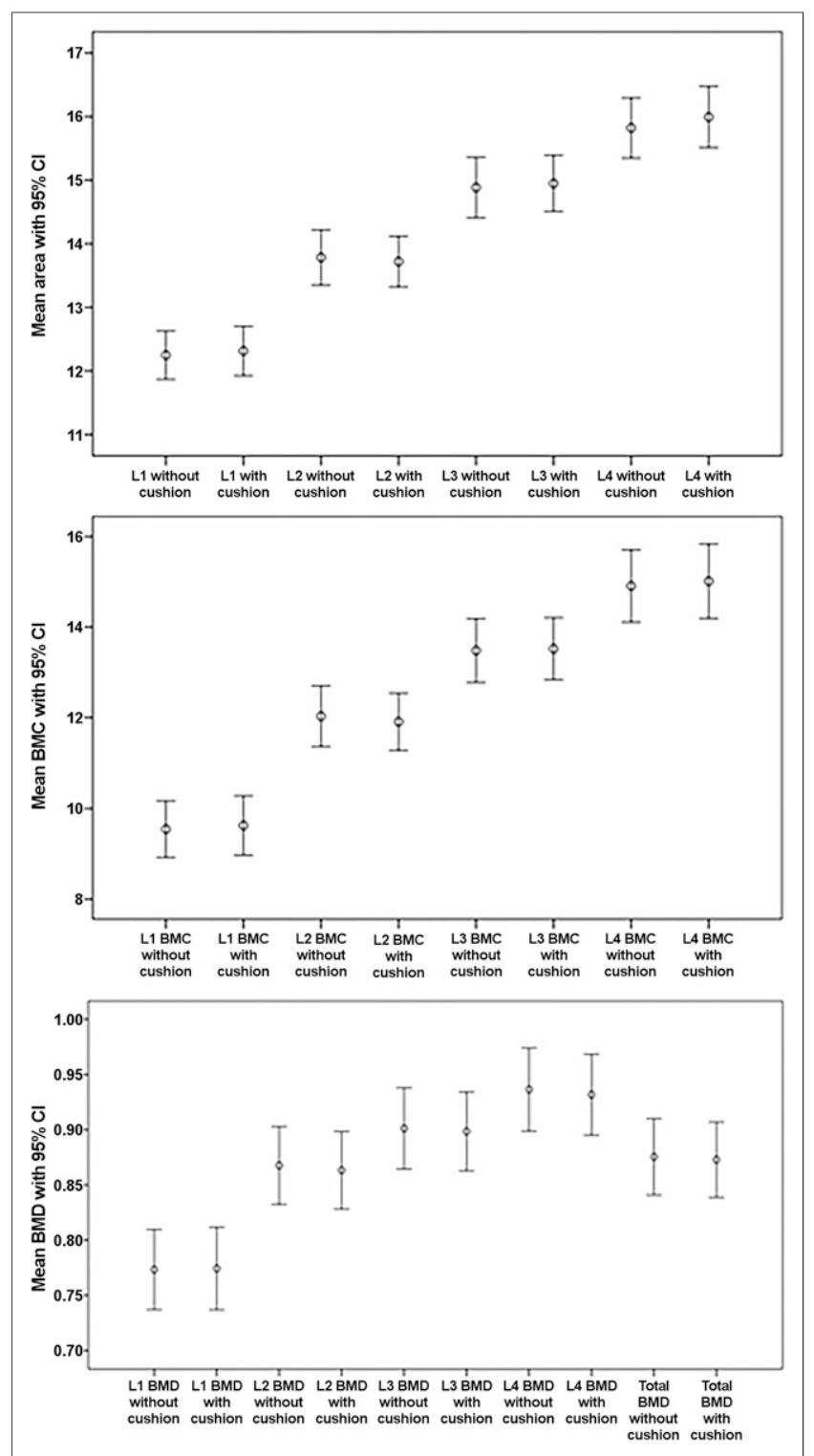

FIGURE 2. Error bar plots of area, bone mineral content (BMC), and BMD. Values gradually increase from $\mathrm{L} 1$ to $\mathrm{L} 4$. $\mathrm{Cl}=$ confidence interval.

hip flexion even without neck flexion. However, we selected a cushion with a fixed height of $8 \mathrm{~cm}$-in other words, a cushion that was not tailored to patient height. The resulting amount of neck flexion attained can generally be considered sufficient to decrease the lordosis.

\section{CONCLUSION}

Use of head positioners to flex the neck and thus minimize lumbar lordosis during dual-energy x-ray absorptiometry does not significantly affect the diagnosis or densitometric measurements from clinical standpoint.

\section{DISCLOSURE}

No potential conflict of interest relevant to this article was reported.

\section{ACKNOWLEDGMENT}

This article was extracted from the postgraduate thesis of Dr. Sajad Ghanbari. The study was conducted at Taleghani Hospital, Shahid Beheshti University of Medical Sciences (SBMU).

\section{REFERENCES}

1. Thorpe JA, Steel SA, Langton CM. A phantom based study on the effect of subject positioning on morphometric $\mathrm{x}$-ray absorptiometry using the Lunar Expert-XL. Br J Radiol. 1998;71:1153-1161.

2. Izadyar S, Golbarg S, Takavar A, Zakariaee SS. The effect of the lumbar vertebral malpositioning on bone mineral density measurements of the lumbar spine by dual-energy X-Ray absorptiometry. J Clin Densitom. 2016;19:277-281.

3. Cheng JC, Sher HL, Guo X, Hung VW, Cheung AY. The effect of vertebral rotation of the lumbar spine on dual energy $\mathrm{X}$-ray absorptiometry measurements: observational study. Hong Kong Med J. 2001;7:241-245.

4. Jeon YK, Shin MJ, Shin YB, et al. Effect of increased axial rotation angle on bone mineral density measurements of the lumbar spine. Spine J. 2014;14:2150-2154.

5. Ikegami S, Kamimura M, Uchiyama S, Nakamura Y, Mukaiyama K, Kato H. Clinical implications of hip flexion in the measurement of spinal bone mineral density. J Clin Densitom. 2016;19:270-276.

6. QDR Reference Manual. Revision 3. Marlborough, MA: Hologic, Inc.; 2009. Document MAN-01016.

7. Dual Energy X Ray Absorptiometry for Bone Mineral Density and Body Composition Assessment. Vienna, Austria; International Atomic Energy Agency; 2011.

8. Pavlovic A, Nichols DL, Sanborn CF, Dimarco NM. Relationship of thoracic kyphosis and lumbar lordosis to bone mineral density in women. Osteoporos Int. 2013;24:2269-2273.

9. Pappou IP, Girardi FP, Sandhu HS, et al. Discordantly high spinal bone mineral density values in patients with adult lumbar scoliosis. Spine. 2006;31:16141620 .

10. Papadakis M, Papadokostakis G, Stergiopoulos K, Kampanis N, Katonis P. Lumbar lordosis in osteoporosis and in osteoarthritis. Eur Spine J. 2009;18:608-613.

11. Papadakis M, Papagelopoulos P, Papadokostakis G, Sapkas G, Damilakis J, Katonis P. The impact of bone mineral density on the degree of curvature of the lumbar spine. J Musculoskelet Neuronal Interact. 2011;11:46-51.

12. Jankowski LG, Warner S, Gaither $\mathrm{K}$, et al. Cross-calibration, least significant change and quality assurance in multiple dual-energy $\mathrm{x}$-ray absorptiometry scanner environments: 2019 ISCD official position. J Clin Densitom. 2019;22:472483

13. Deal CL. Using bone densitometry to monitor therapy in treating osteoporosis: pros and cons. Curr Rheumatol Rep. 2001;3:233-239.

14. Vrtovec T, Pernus F, Likar B. A review of methods for quantitative evaluation of spinal curvature. Eur Spine J. 2009;18:593-607. 